\title{
OPEN Novel structure in the nuclei of honey bee brain neurons revealed by immunostaining
}

\author{
Paul J. Hurd ${ }^{1 凶}$, Kornelia Grübel ${ }^{2}$, Marek Wojciechowski ${ }^{1}$, Ryszard Maleszka ${ }^{3 凶}$ \& \\ Wolfgang Rössler ${ }^{2} \bowtie$
}

In the course of a screen designed to produce antibodies (ABs) with affinity to proteins in the honey bee brain we found an interesting $A B$ that detects a highly specific epitope predominantly in the nuclei of Kenyon cells (KCs). The observed staining pattern is unique, and its unfamiliarity indicates a novel previously unseen nuclear structure that does not colocalize with the cytoskeletal protein f-actin. A single rod-like assembly, 3.7-4.1 $\mu \mathrm{m}$ long, is present in each nucleus of KCs in adult brains of worker bees and drones with the strongest immuno-labelling found in foraging bees. In brains of young queens, the labelling is more sporadic, and the rod-like structure appears to be shorter $(\sim 2.1 \mu \mathrm{m})$. No immunostaining is detectable in worker larvae. In pupal stage 5 during a peak of brain development only some occasional staining was identified. Although the cellular function of this unexpected structure has not been determined, the unusual distinctiveness of the revealed pattern suggests an unknown and potentially important protein assembly. One possibility is that this nuclear assembly is part of the KCs plasticity underlying the brain maturation in adult honey bees. Because no labelling with this $\mathrm{AB}$ is detectable in brains of the fly Drosophila melanogaster and the ant Camponotus floridanus, we tentatively named this antibody AmBNSab (Apis mellifera Brain Neurons Specific antibody). Here we report our results to make them accessible to a broader community and invite further research to unravel the biological role of this curious nuclear structure in the honey bee central brain.

Since the publication of the honey bee draft genome in $2006^{1,2}$, there has been a significant shift in research priorities involving this highly social insect. In particular, a rapid development of molecular tools has facilitated all sorts of genomic, transcriptomics and epigenomic studies ${ }^{3-6}$. However, a limited availability of specific honey bee antibodies has been a critical factor hampering several lines of research that often require a precise localization of a given protein in cells or tissues, in particular those relevant to brain and behaviour. Given the prevalence of antibody usage in detecting specific molecules in vivo and in vitro ${ }^{7}$, this scarcity of antibodies is a potential disadvantage that reduces experimental testability in honey bees versus other invertebrate species like D. melanogaster or C. elegans. Although both the fly and the nematode are gold standards for many areas of biomedical sciences ${ }^{8,9}$, the honey bee with its elaborate social structure and diet-controlled phenotypic polymorphism has much to offer in the study of behaviour and development ${ }^{10,11}$. The three organismal outcomes derived from the same genotype, the two female castes and male drones, are strikingly different in their anatomy, longevity and respective behaviours, and their brains show remarkable structural plasticity during adult behavioural maturation ${ }^{12-15}$. In recent years, the honey bee emerged as a convenient system in which the interactions between gene regulatory networks involving DNA-binding proteins and the epigenomic decorations associated with DNA methylation can be analysed ${ }^{16,17}$. This important aspect of cellular regulation cannot be investigated in flies and nematodes that lost the DNA methylation enzymology and cannot provide meaningful input into the function of epigenomic communication systems $s^{4,18}$.

This project is part of our interest in identifying and characterising molecules controlling cellular processes in the brain and ultimately behaviour via a set of mechanisms broadly referred to as epigenetic ${ }^{19}$. An important aspect of this line of research is the development of antibodies to advance functional classification of proteins predicted to be relatives of the mammalian epigenetic toolkit. The honey bee proteome is mostly annotated by

\footnotetext{
${ }^{1}$ School of Biological and Chemical Sciences, Queen Mary University of London, London E1 4NS, UK. ${ }^{2}$ Behavioral Physiology and Sociobiology (Zoology II), Biozentrum, University of Würzburg, Am Hubland, 97074 Würzburg, Germany. ${ }^{3}$ Research School of Biology, The Australian National University, Canberra, ACT 2601, Australia. ${ }^{\boxplus e m a i l: ~}$ p.j.hurd@qmul.ac.uk; ryszard.maleszka@anu.edu.au; roessler@biozentrum.uni-wuerzburg.de
} 
comparative analyses using datasets of model species. However, many important differences between various lineages suggest that functional transferability based exclusively on sequence comparisons is not sufficient to imply species-specific roles ${ }^{10,20,21}$. It is now clear that data transfer between the genome/epigenome, transcriptome and phenome needs to include the co- and post-translational levels and antibody-based molecular anatomy ${ }^{22}$. Here we report a characterisation of a newly produced antibody AmBNSab that detects an unusual nuclear structure predominantly in the intrinsic neurons (Kenyon cells) of the honey bee mushroom bodies (MBs), a prominent bilateral neuropil found in the brains of most arthropods ${ }^{23}$. Some features of the observed immunostaining pattern suggest that this nuclear structure is not only novel but might be specific to the honey bee.

\section{Results and discussion}

Our initial aim was to generate an antibody against the single honey bee relative of mammalian ten-eleven translocation methylcytosine dioxygenases (TETs) ${ }^{24}$, a family of proteins implicated in numerous cellular activities including DNA demethylation and cooperative regulation of gene networks ${ }^{25}$. As it often happens, an antibody produced to bind to one protein can bind to another ${ }^{26}$, and as shown in this case, with excellent affinity. The result of a Western blot shown in figure S1A indicates that this new $\mathrm{AB}$ detects a large $>300 \mathrm{kD}$ protein in the brain extract of adult honey bee foragers. Only a single and clear band is visible suggesting that the antibody has a high level of specificity for only one protein expressed in the brain. However, the antibody failed to detect the

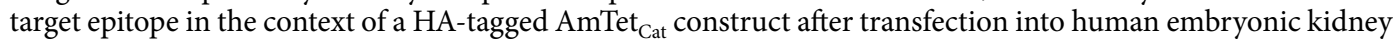
(HEK) 293 cells (Figure S1B). Given this result, the antibody was deemed to be novel, and subsequently named AmBNSab (see below). Following the initial validation, we performed high-resolution microscopy to examine the intracellular localisation of AmBNSab. We used adult brains dissected from two female castes, workers, and queens, as well as from males (drones). The drones have quite unusual brain anatomy dominated by huge optic lobes with the mushroom bodies somewhat hidden underneath. In addition, we examined the AmBNSab labelling pattern in preadult stages, namely larvae of various ages, and stage 5 pupae ${ }^{27}$. The aim of this experiment is to determine if the new nuclear structure is present before and through metamorphosis, during which the nervous system is reorganised to replace most larval neurons with adult neurons ${ }^{28}$.

Figure 1 shows an overview and the details of AmBNSab immunolocalization in the brain of adult honey bee workers. The overview reveals an evenly distributed light background fluorescence in all parts of brain (Fig. 1A). At high magnification, AmBNSab-labelled rod-like structures are clearly visible in nuclei of Kenyon cells (KCs), the intrinsic neurons of the $\mathrm{MB}$ a large proportion of which are housed within the cup shaped MB calyx (Fig. 1B,D,E,G). This applies to the inner compact, inner non-compact (class I) and the group of outer (class II) KC nuclei (Fig. 1C,E). We estimate the rods to be 3.7-4.1 $\mu \mathrm{m}$ in length in workers and drones and $\sim 2.1 \mu \mathrm{m}$ in young queens. Figure 2 shows examples of $3 \mathrm{D}$ reconstructions of individual KC nuclei from the group of inner non-compact KCs. The average length of rod-like assemblies obtained from randomly chosen KCs within individual worker bees (foragers, $\mathrm{n}=10$ in each case) was $3.7 \mu \mathrm{m}$ for the class I inner compact KCs, $4.1 \mu \mathrm{m}$ in the class I inner non-compact $\mathrm{KC}$, and $3.6 \mu \mathrm{m}$ in the class II outer $\mathrm{KCs}$, which roughly correlates with the different size of the KCs in the three groups (for the spatial arrangement and characteristics of different KC groups, see Fig. 1 in Groh and Rössler ${ }^{29}$. The rods are positioned across the middle, but do not seem to be attached to the nuclear envelope (Figs. 1H, 2). Labelling is mostly absent or negligible in cell nuclei of other brain regions. For example, only a few neurons in the antennal lobes show labelling with AmBNSab (Fig. 1F,I) with virtually no signal detectable in other parts of the brain. To determine if AmBNSab detects a similar structure in other insects, we analysed brains of the fly Drosophila melanogaster and the carpenter ant, Camponotus floridanus. We were interested to examine if this novel structure is present in other insects, or perhaps is restricted to Hymenoptera, or even to one family of Apidae. As shown in Fig. 3, AmBNSab labelling is found in KCs of the honey bee with no binding detectable in KCs of both the fly and ant. To determine if the expression of this unusual nuclear structure is temporally regulated, we examined the AmBNSab labelling pattern in the larval and pupal stages. The results shown in Fig. 4 demonstrate that no labelling is detectable in both early (Fig. 4A,E) and late larvae (Fig. 4B,F). Interestingly, during pupal metamorphosis and a critical phase of brain development at pupal stage 5 , only weak and sporadic labelling is visible around the cell nucleus (Fig. 4C,G). In contrast, in brains of young 1-day old workers, the structures with a typical rod-like shape are already visible, although they are shorter and not as frequent as in older brains (Fig. 4D,H). Although the labelling is well-defined in both female castes and drones, the rods are shorter in young queens (Fig. 5A,B) and more comparable to very young workers (Fig. 4D,H) than to adult workers or drones (Fig. 5C,D,E,F). In drones the rod-like assemblies were on average $3.6 \mu \mathrm{m}$ long in the compact inner KCs, and $4.0 \mu \mathrm{m}$ in the non-compact inner KCs, whereas in young virgin queens the average length in the compact and non-compact inner KCs was $2.1 \mu \mathrm{m}(\mathrm{n}=10$ in each case; in young queens, the variability was greater in comparison with foragers). To determine if the AmBNSab binding pattern colocalizes with the cytoskeletal protein $\mathrm{f}$-actin, we used a combined AmBNSab and f-actin with phalloidin labelling (Fig. 6A,D). The choice of $\mathrm{f}$-actin was motivated by recent evidence that in addition to its cytoplasmic function, this protein also has a nuclear role in organizing chromatin during mitosis. The labelling patterns shown in Fig. 6 rule out any spatial association between the rod-like structures and $\mathrm{f}$-actin in the KC nuclei. To test for possible interferences between secondary antibodies and chromatin markers, two different staining combinations for cell nuclei (Hoechst 34580 and Sytox green) were combined with AmBNSab labelling. Both patterns reveal a very similar result regarding the rod-like assemblies (Fig. 6A,D). In addition, no related structures were detected in the chromatin labeling (for more details on all images see Figs. 1, 2, 3, 4, 5, 6 legends).

Taken together, our immunolocalization images show a unique and distinctly arranged protein assembly predominantly in intrinsic neurons of the MBs in adult honey bee brains. The intensity of immunostaining during postembryonic development is variable with no or very little labelling detectable during larval and pupal stages, and strong labelling seen in adult brains of worker bees, especially foragers, and drones, but weaker in 

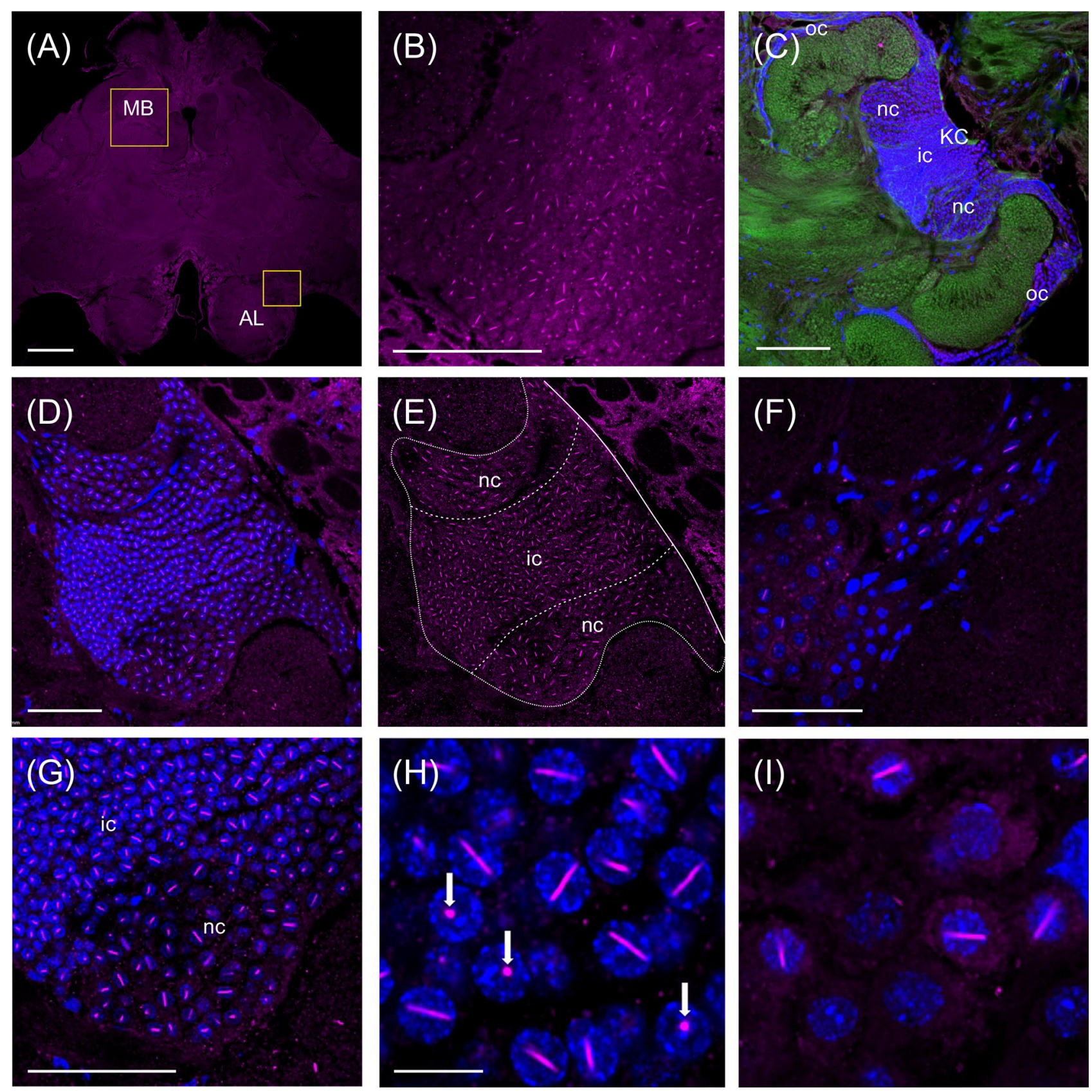

Figure 1. Immunolocalization of AmBNSab in the brain of adult honey bee workers. (A) Overview of the honey bee brain labeled with AmBNSab (magenta) at low magnification shows evenly distributed low background in most parts of the brain. (B) At higher magnification AmBNSab specific labeling in the mushroom body (MB) calyx (area defined by the box in a) reveals rod-like assemblies in KC nuclei. (C) Detail of one MB calyx double labeled with Hoechst 34580 (blue) and f-actin phalloidin (green) as orientation for the following figures. The localization of inner compact (ic), inner non-compact (nc) and outer compact (oc) Kenyon cells (KC) is indicated. (D,E) Distinct labeling with AmBNSab in KC nuclei in the MB calyx cup. Double labeling with Hoechst 34580 (blue) and AmBNSab (magenta) in (D) and AmBNSab channel only (magenta) in (E). The lines in (E) demarcate the border between the KC nuclei belonging to the inner compact and non-compact KC nuclei. (G,H) Double stained KC nuclei at higher magnification. Labeling with antiAmBNSab (magenta) and cell nuclei staining with Hoechst 34580 (blue). Overview of the inner compact and non-compact $(\mathbf{G})$ and high magnification of non-compact KC nuclei in $(\mathbf{H})$. Both KC types contain AmBNSab positive rod-like structures with variable spatial orientation $(\mathbf{G}, \mathbf{H})$. The arrows in $(\mathbf{H})$ indicate KC nuclei where the rod-like assemblies are viewed in a cross section. (F,I) In contrast to the MBs, the antennal lobe (AL) and other parts of the brain (not shown) contain no or much smaller numbers of cell nuclei with AmBNSab positive rod-like structures. Overview of cell nuclei in a cluster of AL neurons (F), shown at higher magnification in (I). Only few somata contain cell nuclei with AmBNSab positive labeling. Scale bars: $\mathbf{A}=200 \mu \mathrm{m}, \mathbf{B}=50 \mu \mathrm{m}$, $\mathbf{C}=100 \mu \mathrm{m}, \mathbf{D}, \mathbf{E}=50 \mu \mathrm{m}, \mathbf{F}=50 \mu \mathrm{m}, \mathbf{G}=50 \mu \mathrm{m}, \mathbf{H}-\mathbf{I}=10 \mu \mathrm{m}$. Five brains were analysed. 
(A)

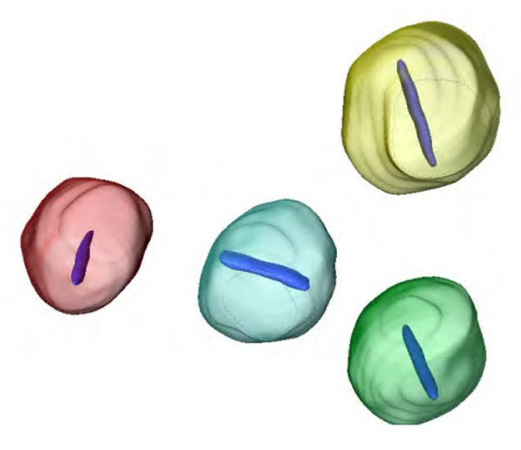

(B)

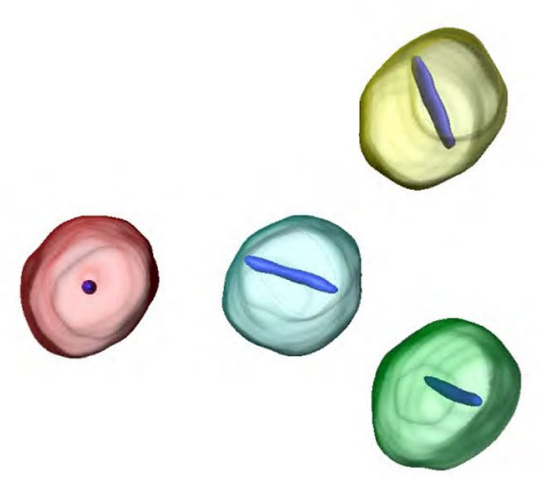

scale: $5 \mu \mathrm{m}$

(C)

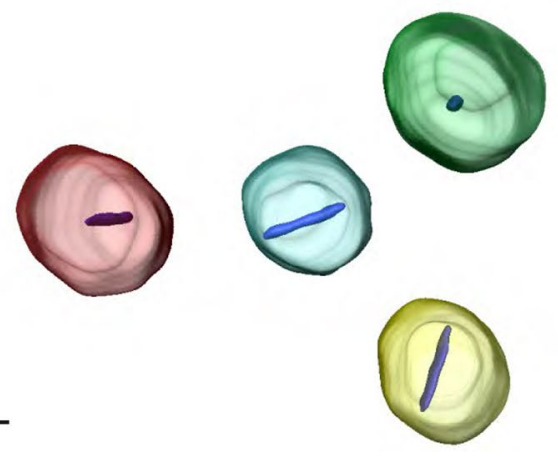

Figure 2. 3D-reconstructions of individual Kenyon cell (KC) nuclei containing the rod-like assembly of AmBNSab labeling. (A-C) Random selection of 4 different KC nuclei from the group of inner non-compact KCs shown in different orientations. Each nucleus is shown in a different colour. The average length $(\mathrm{N}=10)$ of the rod-like assemblies (blue) in this group of KCs is $4.1 \mu \mathrm{m}$. Scale bar $=5 \mu \mathrm{m}$.

young queens and 1-day-old workers. Such context-dependent expression is consistent with a potential biological role of this novel structure in the mushroom bodies (MBs), an important neuropil implicated in multimodal sensory processing, integration, associative learning, and memory formation ${ }^{23,30-32}$. One important feature of the $\mathrm{MB}$ neuropil is its volumetric plasticity in the absence of neurogenesis ${ }^{13-15,33-37}$. These volumetric changes and structural plasticity of synaptic complexes in the MBs occur during adult maturation of honey bees and are driven by both age and experience. In this context, our finding that this novel structure becomes more pronounced in older foraging bees suggests a possible connection to $\mathrm{MB}$ plasticity, and previously unexplored aspects of 3D architecture in the nuclei of Kenyon cells. Since the brain cells are postmitotic in adult honey bees ${ }^{34}$, the rod-like structure is mostly built after emergence in non-dividing KCs. It is noteworthy that cell cycle proteins are active in non-dividing neurons and their role in synaptic plasticity has been highlighted in several studies ${ }^{38}$. Axonal elongation, dendrite morphogenesis and synaptic maturation are all part of synaptic plasticity. While at this stage the connection between the novel nuclear structure and $\mathrm{KC}$ plasticity is purely speculative, the postmitotic dynamics of these neurons offers a promising avenue for further investigations.

While the lack of cross-species labelling is interesting, it is not unusual because lineage-specific gene inventions are quite common amongst insects ${ }^{39-44}$. Indeed, predicted proteins with no relatives in other species represent a functional void that can only be filled by multilevel analyses including antibody research. Alternatively, 

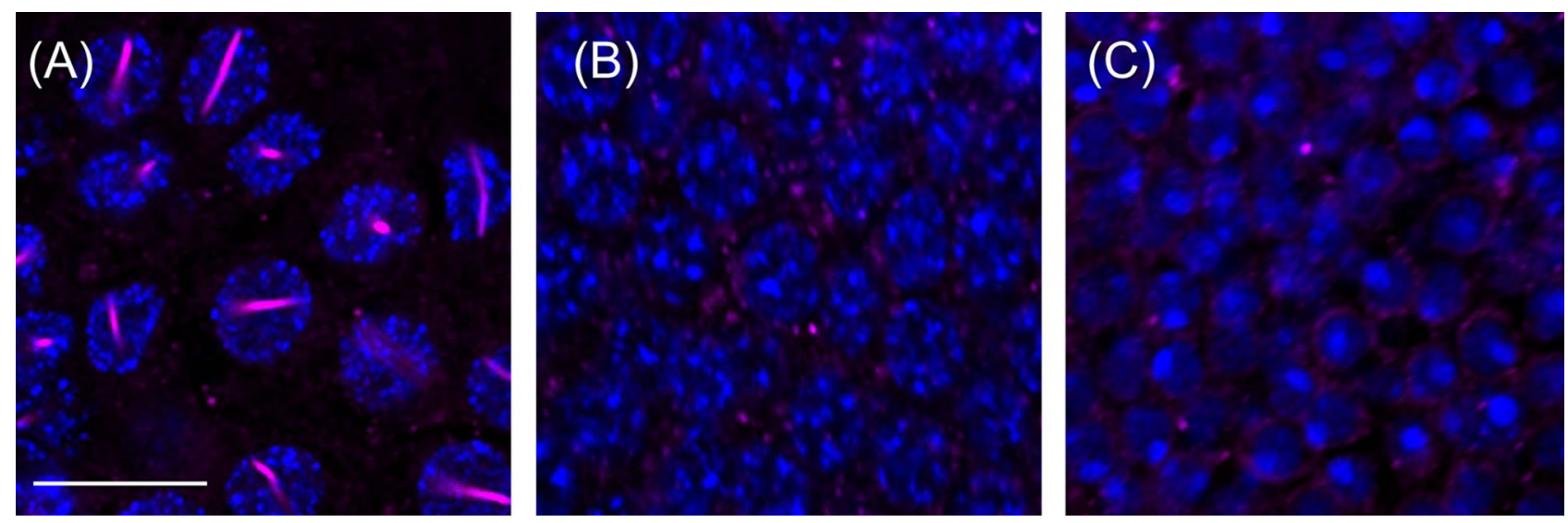

Figure 3. Labeling of rod-like assemblies in Kenyon cell (KC) nuclei with AmBNSab is specific in honey bee brains. (A-C) Comparison of labeling of KC nulcei in the mushroom body of honeybee (Apis mellifera) worker (A) with labeling in the carpenter ant, Camponotus floridanus (B) and the fruit fly, Drosophila melanogaster (C), all shown at the same magnification. KC nuclei labeled with Hoechst 34580 in blue and staining with AmBNSab in magenta. Rod-like structures are present only in $\mathrm{KC}$ nuclei of the honey bee brain. Scale bar in a, also valid for $\mathbf{B}, \mathbf{C}=10 \mu \mathrm{m}$. Number of brains analysed: A. mellifera $=2$, C. floridanus $=4 ; \mathbf{D}$. melanogaster $=4$.
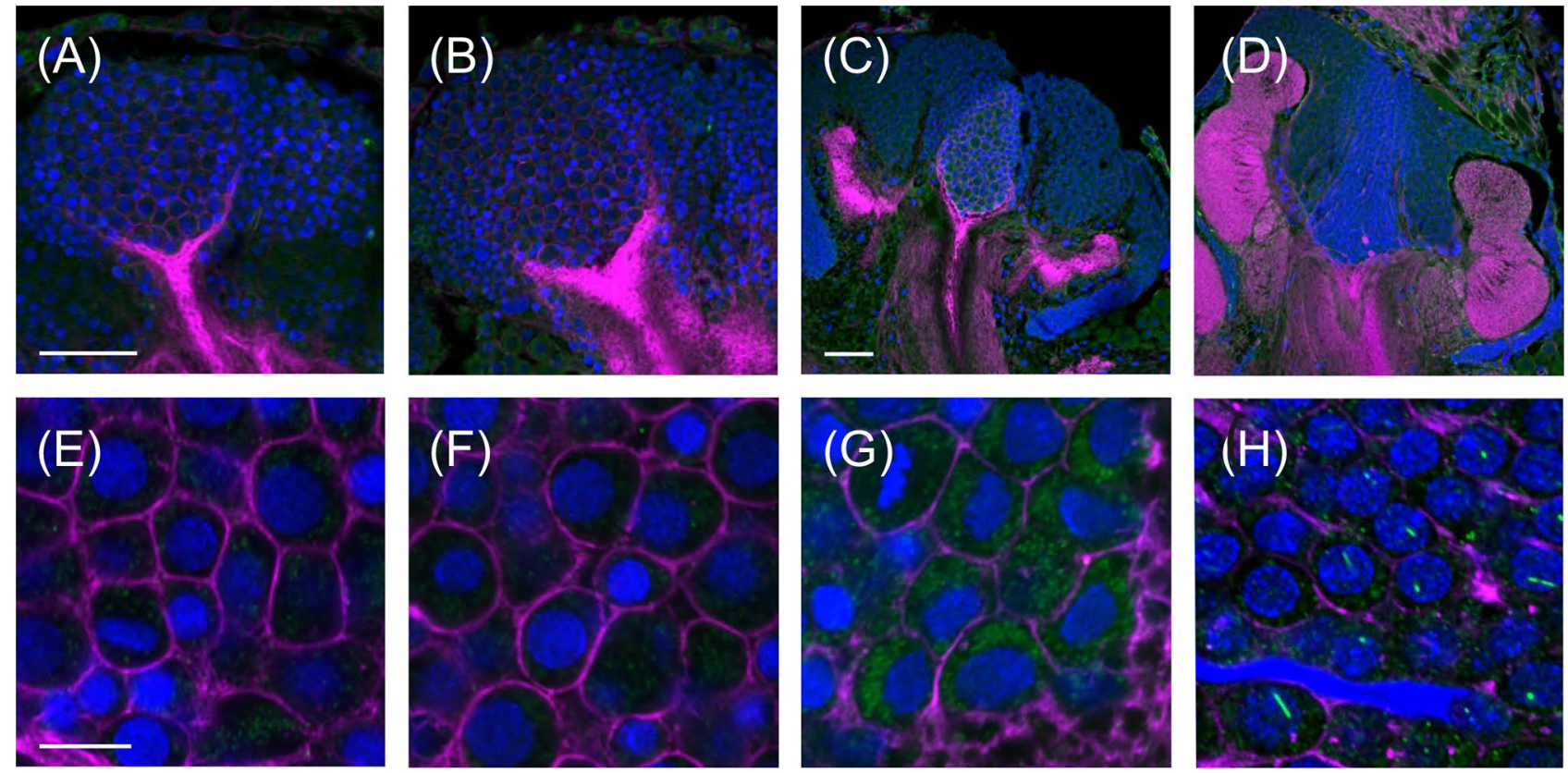

Figure 4. (A-H) Life-stage specific differences in labeling of Kenyon cell (KC) nuclei with AmBNSab in honey bees. Staining of cell nuclei with Hoechst 34580 (blue), f-actin phalloidin (magenta) and AmBNSab (green). (A-H) Images are shown at two different magnifications with an overview of the mushroom bodies (MBs) (top row) and details of KC nuclei at higher magnification (bottom row). Labeling with AmBNSab was absent at early larval stages $(\mathbf{A}, \mathbf{E})$, late larval stages $(\mathbf{B}, \mathbf{F})$. At pupal stage 5 , during the peak of postembryonic brain metamorphosis, only weak and sporadic labeling is visible in the cytosol of $\mathrm{KCs}(\mathbf{C}, \mathbf{G})$. Labeling with AmBNSab in KCs of day 1 adult workers revealed typical rod-like assemblies in KC nuclei, although more sporadic and with shorter rod lengths compared to adult foragers (D,H). Scale bars in $\mathbf{A}$ (also for $\mathbf{B}$ ) and $\mathbf{C}$ (also for $\mathbf{D})=50 \mu \mathrm{m}$, in $\mathbf{E}$ (also for $\mathbf{F}-\mathbf{H})=10 \mu \mathrm{m}$. Number of brains analysed: foragers $=2$, early larvae $=5$, late larvae $=5$, stage 5 pupae $=5,1$-day old adults $=4$.

a related protein is present in these insects, but with sufficiently altered sequence preventing AmBNSab binding. At this stage, the target protein detectable by AmBNSab remains in the category of uncategorised, possibly honeybee-specific proteins, that needs to be further characterised. Moving from a cellular description of where and when this protein is found to biological function will need to incorporate a far greater component of cuttingedge biochemistry, including immunoprecipitation, peptide sequencing and cell sorting. We are confident that this goal can be promptly achieved, and our antibody will soon become a valuable tool in the study of brain and behaviour. 

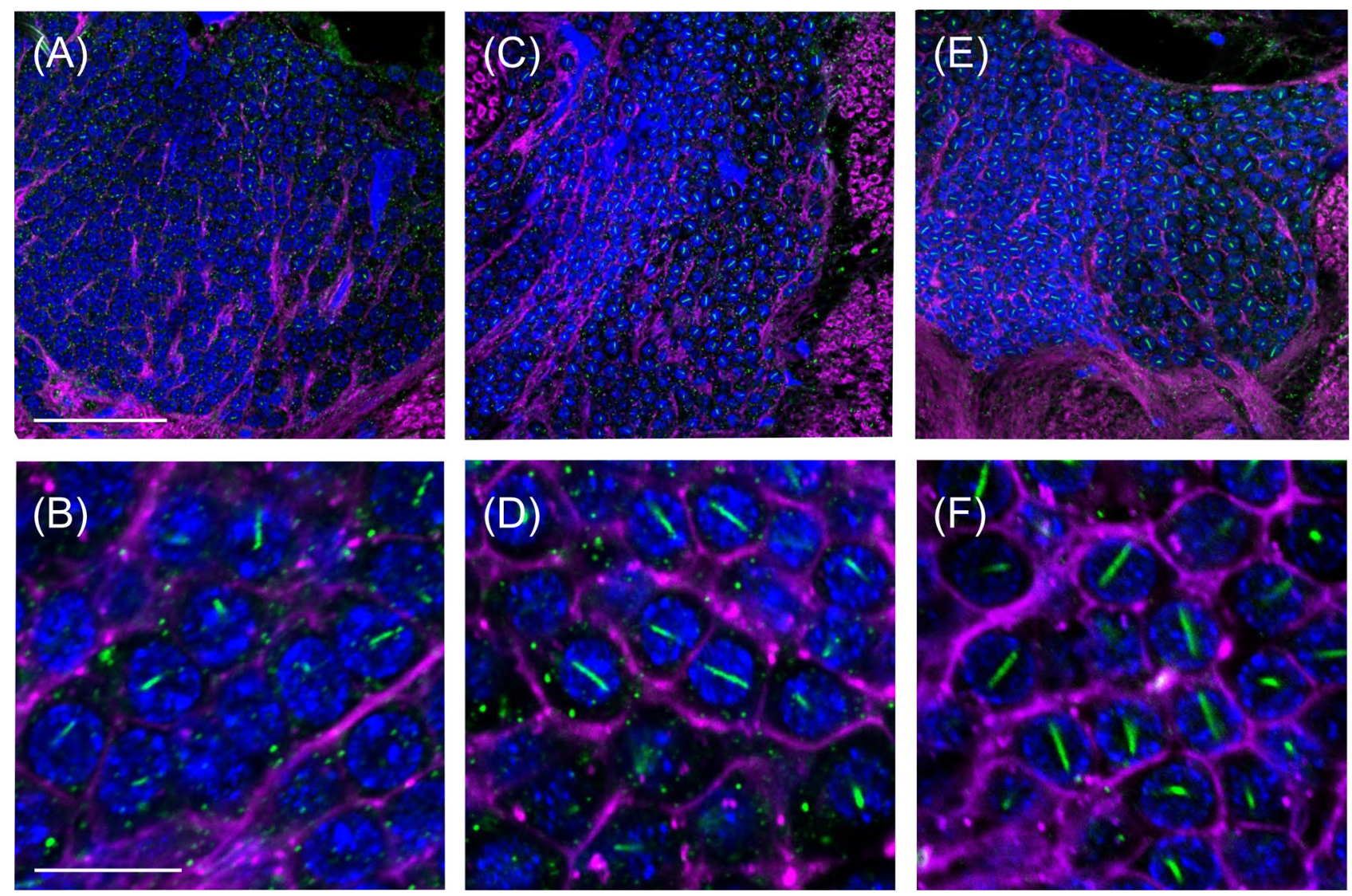

Figure 5. Caste- and sex-specific differences in labeling with AmBNSab. Staining with Hoechst 34580 (blue), f-actin phalloidin (magenta), and AmBNSab (green). (A,B) Example in a young (1-day old) virgin queen shows relatively short, rod-like assemblies in Kenyon cell (KC) nuclei labeled with AmBNSab compared to experienced female workers (foragers, E-F). (C-D) In the brain of a male (drone) rod-like assemblies in KC nuclei labeled with AmBNSab are comparable to those found in adult female foragers (E-F). (E-F) Positive control in an adult (female) worker bee (forager) shows typical rod-like assemblies of AmBNSab within different orientation in individual KC nuclei. Scale bars upper row (in $\mathbf{A}$ ) $=50 \mu \mathrm{m}$, lower row (in B) $10 \mu \mathrm{m}$. Number of brains analysed: foragers $=10$, young virgin queens, $2-4$ days-old $=3$, drones (unknown age) $=3$.

\section{Conclusion}

We uncovered an intriguing nuclear structure in the Kenyon cells of the honey bee brain. Although the significance of this structure remains to be established, its context-dependent expression is suggestive of a role in nuclear architecture and possibly, the adult brain volumetric plasticity. This unexpected finding clearly shows that previously unexplored 3-D features of neurons can be visualised with powerful molecular tools including highly specific antibodies. An important aspect of this study is our demonstration that the nucleus still holds surprises that can be revealed with advanced bioimaging.

\section{Materials and methods}

Antibody generation. Initially we aimed to produce antibodies against the honey bee relative of mammalian TETs (Ten-eleven translocation methylcytosine dioxygenases) by targeting highly conserved catalytic regions of the predicted honey bee TET splice variants ${ }^{24}$ with the construct used to obtain the crystal structure of human TET2 (PDB: 4NM6) ${ }^{45}$ using MUSCLE (MUltiple Sequence Comparison by Log-Expectation). This alignment was used to generate a cropped consensus splice variant encompassing the structurally characterised catalytic domain of AmTET ${ }^{24}$. This sequence was analysed using peptide immunogenicity prediction software at Eurogentec (Liege, Belgium). Two of the suggested peptides were selected (CLRRSGLEEKILTIVK and CVVTMTKHRTLSKPED, C-terminally amidated) for synthesis and further immunisation at Eurogentec. In total, two rabbits were immunised with a combination of both peptides using a 28-day protocol. Rabbit immunisation was done under the strict rules of Eurogentec that complies with the highest standards for animal welfare, including the Federation of European Laboratory Animal Science Associations (FELASA), the Belgian Accreditation Body (BELAC), and the UK Home Office Animals Scientific Procedures Act. Ethical approval of the work was obtained from the EU Animal Experimentation Ethics Committee (Protocol Number A2016/12).

Western blotting. Adult worker brains were homogenised in PBS containing cOmplete protease inhibitor cocktail (Roche) using a plastic pestle. An equal volume of SDS-PAGE loading buffer was then added, and 

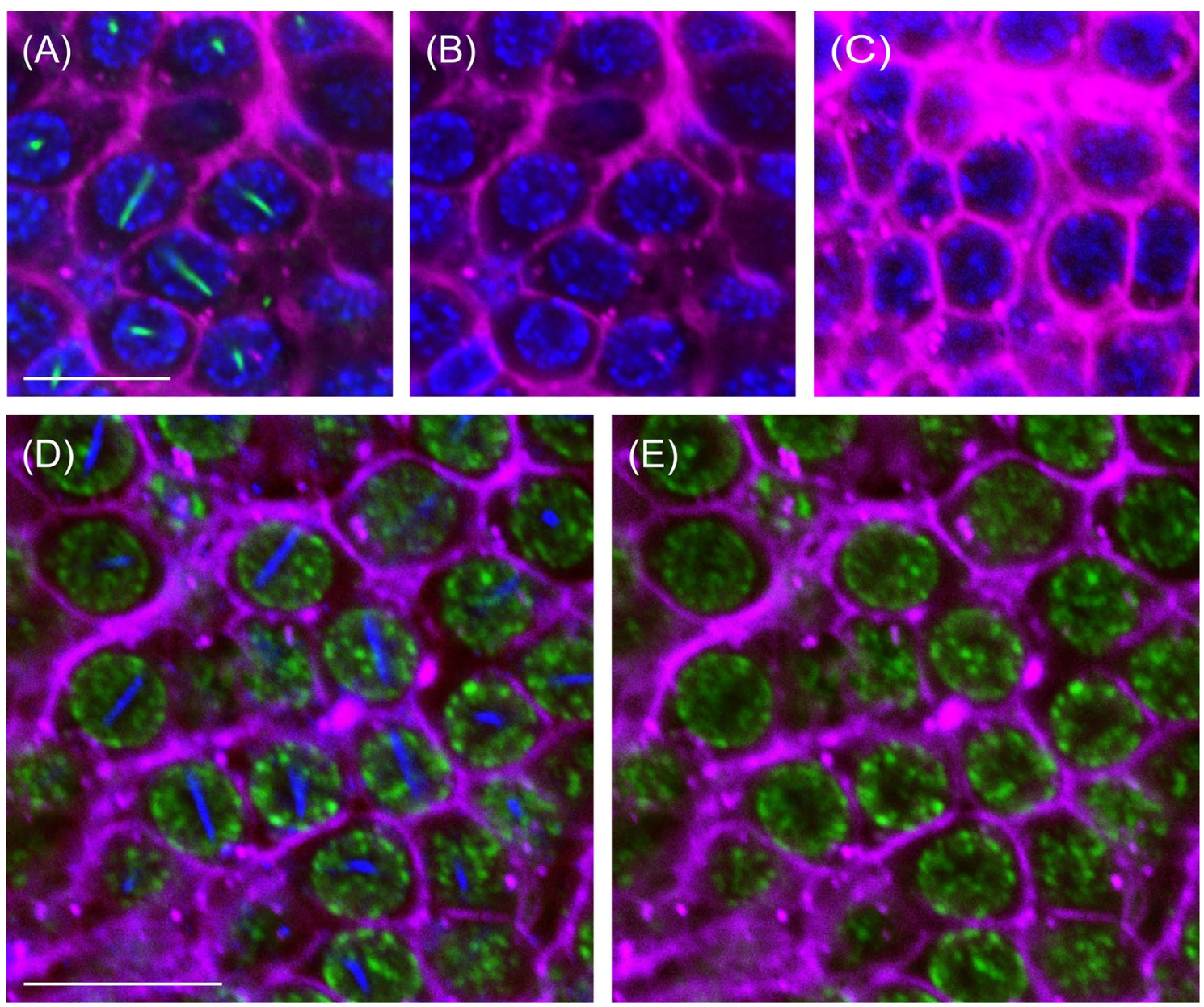

Figure 6. High magnification analyses of Kenyon cells (KCs) double labeled with AmBNSab and f-actin phalloidin in the brain of honey bee workers. (A-C) Labeling of KC nuclei with Hoechst 34580 (blue), f-actin phalloidin (magenta) and AmBNSab (green). Triple labeling in (A) shows that rod-like assemblies of AmBNSab do not colocalize with $\mathrm{f}$-actin phalloidin. F-actin phalloidin labeled structures $(\mathbf{B})$ do not reveal any structure related to the AmBNSab positive rod-like assemblies in (A). Strong excitation of the $\mathrm{f}$-actin phalloidin (magenta) (C) suggests that the rod-like assemblies of AmBNSab (A) do not have any obvious association with the f-actin cytoskeleton inside the cell nucleus. (D-E) Triple labeling of KCs with Sytox green (green), f-actin phalloidin (magenta) and AmBNSab (blue). The rod-like assemblies of AmBNSab within Sytox-green labeled cell nuclei are clearly visible (D), and neither Sytox green, nor f-actin phalloidin labeling (E) shows any obvious association with the rod-like assemblies of AmBNSab labelling (D). Scale bar in (A) for upper row $=10 \mu \mathrm{m}$, in (D) for lower row $=10 \mu \mathrm{m}$. Number of forager brains analysed: with Hoechst $34580=3$, with Sytox Green $=3$.

samples were heated to $95^{\circ} \mathrm{C}$ and incubated for $5 \mathrm{~min}$. Samples were then cleared by a $5 \mathrm{~min}$, full-speed centrifugation in a microcentrifuge. Cleared lysates were loaded onto a $6 \%$ SDS-PAGE gel and resolved until the dye migrated out of the gel. Afterwards, the resolved gel was blotted onto a Protran nitrocellulose membrane (GE Healthcare). Transfer was carried out for $16 \mathrm{~h}$ at $30 \mathrm{~V} / 90 \mathrm{~mA}$ in CAPS transfer buffer (10 mM Na-CAPS, 10\% methanol; pH 11) using a Mini Trans-Blot chamber (Bio-Rad). Membranes were blocked with 5\% skim milk dissolved in PBST for $1 \mathrm{~h}$ at RT. Immune sera were diluted 1:500 in the blocking solution and incubated with the membrane overnight at $4^{\circ} \mathrm{C}$. The membrane was then washed three times for $10 \mathrm{~min}$ with PBST. Anti-rabbit IgG HRP conjugated secondary antibody (\#31,462, Thermo Fisher) was diluted 1:30,000 in blocking solution and incubated with the membrane for $1 \mathrm{~h}$ at $4{ }^{\circ} \mathrm{C}$ and washed three times for $10 \mathrm{~min}$ with PBST. Blot was developed using Pierce ECL substrate (ThermoFisher) and visualised with a Biorad ChemiDoc Touch Imaging System.

Antibody validation with heterologously expressed HA-tagged Tet proteins. Cloning of the HA-tagged catalytic domains of $A$. mellifera and $H$. sapiens Tet proteins into a pcDNA3 mammalian overexpression vector has been described previously ${ }^{24}$. To determine if the new antibodies recognised AmTet, HEK293 cells were grown to 50-60\% confluency in a 6-well plate and transfected with $1.5 \mu \mathrm{g}$ of plasmid DNA per well, using a standard PEI protocol. After $24 \mathrm{~h}$, cells were washed twice with PBS and harvested. Collected cells were suspended in SDS-PAGE loading buffer containing cOmplete protease inhibitor cocktail (Roche), and heat-lysed 
at $95{ }^{\circ} \mathrm{C}$ for $5 \mathrm{~min}$. Lysates were cleared by centrifugation and loaded onto an $8 \%$ SDS-PAGE gel. Subsequent western blotting was carried out as described earlier.

Animals. Different life stages of honey bee (Apis mellifera carnica) workers, young virgin queens, and drones were obtained from colonies reared at the institutional (Zoology II) apiary at the Biocenter, University of Würzburg, Germany. Staging of larvae and pupae was done according to Groh and Rössler ${ }^{27}$. For all neuroanatomical procedures, bees were anaesthetized on ice and then mounted for preparation.

Immunohistochemistry. This protocol is described in more detail in our previous work ${ }^{27,35}$. For brain dissections, heads, or the entire bodies in the case of larvae and pupae, were covered with physiological saline (130 mM NaCl, $5 \mathrm{mM} \mathrm{KCl}, 4 \mathrm{mM} \mathrm{MgCl}, 5 \mathrm{mM} \mathrm{CaCl}_{2}, 15 \mathrm{mM}$ Hepes, $25 \mathrm{mM}$ glucose, $160 \mathrm{mM}$ sucrose; $\mathrm{pH}$ 7.2). A window was cut in the head, and glands, tracheae and the pharynx were removed. Brains were immediately transferred into ice-cold 4\% formaldehyde (methanol free, 28,908, Fischer Scientific, Schwerte, Germany) in phosphate-buffered saline (PBS; $\mathrm{pH} 7.2$ ) and fixed at $4{ }^{\circ} \mathrm{C}$ overnight. Fixed brains were washed with PBS, embedded in 5\% low-melting point Agarose (Agarose II, no. 210-815, Amresco, Solon, OH) and sectioned in frontal planes at $80-100 \mu \mathrm{m}$ using a vibrating microtome (Leica VT 1000S, Nussloch, Germany). Sections were washed in PBS with $2 \%$ Triton-X $100(1 \times 10 \mathrm{~min})$, PBS with $0.2 \%$ Triton-X $100(2 \times 10 \mathrm{~min})$ and pre-incubated in PBS with $0.2 \%$ Triton-X 100 and $2 \%$ normal goat serum (NGS, DIANOVA GmbH, Hamburg, Germany) for $1 \mathrm{~h}$ at room temperature on a shaker. Afterwards, sections were incubated in AmBNSab antibody (rabbit) (1:1000) in PBS with $0.2 \%$ Triton-X 100 and $2 \%$ NGS for 2 days at $4{ }^{\circ} \mathrm{C}$. Then, sections were incubated in secondary antibody (1:250, Alexa Fluor 488 and 405, goat anti rabbit, Kat.No. A-11008 and A-31556, Invitrogen by Thermo Fisher Scientific, Carlsbad, CA, USA) in PBS with 1\% NGS. To label f-actin, CF633 Fluor conjugated Phalloidin (0.2 units, Kat.-Nr. 00,046, Biotium, Hayward, CA, USA) was added, incubated over night at $4{ }^{\circ} \mathrm{C}$ and then rinsed in PBS $(2 \times 10 \mathrm{~min})$. For cell nuclei labelling, preparations were incubated in Hoechst 34580 (1:1000, Kat.No. H21486, LifeTechnologies GmbH, Darmstadt, Germany), or, in some cases, in Sytox Green (1:1000, Kat.No. S7020; LifeTechnologies GmbH, Darmstadt, Germany) in PBS for $15 \mathrm{~min}$ at room temperature, and then rinsed in PBS (4×10 min). Finally, sections were transferred in 60\% Glycerol in PBS for $30 \mathrm{~min}$ and mounted on slides in $80 \%$ Gylcerol in PBS. Cover slips were sealed with nail polish and preparations kept in the dark at $4{ }^{\circ} \mathrm{C}$ until microscopy or $-20^{\circ} \mathrm{C}$ for long-term storage.

Laser scanning confocal microscopy and 3D-image processing. Brain samples were scanned with a confocal laser scanning microscope (Leica TCS SP2 and SP8, Leica Microsystems AG, Wetzlar, Germany) using a $20 \times$ or $63 \times$ water immersion objective $(20.0 \times / 0.7 \mathrm{NA}$ or $63 \mathrm{x} / 1.20 \mathrm{NA})$. Additional digital zoom was applied during image acquisition. Preparations were excited at wavelengths of 405 (for Hoechst 34580), 488 (for Sytox green and CF488), 568 (for A568) and 633 (for CF633) depending on the fluorophores used. All samples were scanned at a resolution of 1,024 $\times 1,024$ pixels in xy-direction. For 3D image stacks, samples were scanned at $\mathrm{z}$-step size of $0.5 \mu \mathrm{m}$. Single images or image stacks were processed using ImageJ (ImageJ 1.52p; Wayne Rasband, NIH, Bethesda, MD) and CorelDrawX9 (Version 21.0.0.593, Corel Corporation, Ottawa, OB, Canada). If necessary, contrast was adjusted in ImageJ or Corel Draw. Three-dimensional reconstructions of confocal image stacks were generated using the software Amira 2019.1 (FEI, Visualization Sciences Group; Hillsboro, OR; http://thermofisher.com/amira-avizo). The 3D surface models were generated using the SurfaceGen module.

Received: 7 October 2020; Accepted: 10 March 2021

Published online: 25 March 2021

\section{References}

1. Weinstock, G. M. et al. Insights into social insects from the genome of the honeybee Apis mellifera. Nature 443, 931-949. https:// doi.org/10.1038/Nature05260 (2006).

2. Robinson, G. E. et al. Sweetness and light: illuminating the honey bee genome. Insect. Mol. Biol. 15, 535-539. https://doi.org/10. $1111 /$ j.1365-2583.2006.00698.x (2006).

3. Wojciechowski, M. et al. Phenotypically distinct female castes in honey bees are defined by alternative chromatin states during larval development. Genome Res. 28, 1532-1542. https://doi.org/10.1101/gr.236497.118 (2018).

4. Maleszka, R. Epigenetic code and insect behavioural plasticity. Curr. Opin. Insect Sci. 15, 45-52. https://doi.org/10.1016/j.cois. 2016.03.003 (2016).

5. Kucharski, R. \& Maleszka, R. Exploring DNA methylation diversity in the honey bee brain by ultra-deep amplicon sequencing. Epigenomes 4, 10 (2020).

6. Feil, R. \& Fraga, M. F. Epigenetics and the environment: emerging patterns and implications. Nat. Rev. Genet. 13, 97-109. https:// doi.org/10.1038/Nrg3142(2012).

7. Goldman, R. D. Antibodies: indispensable tools for biomedical research. Trends Biochem. Sci. 25, 593-595. https://doi.org/10. 1016/s0968-0004(00)01725-4 (2000).

8. Jennings, B. H. Drosophila-a versatile model in biology and medicine. Mater. Today 14, 190-195. https://doi.org/10.1016/S13697021(11)70113-4 (2011).

9. Kaletta, T. \& Hengartner, M. O. Finding function in novel targets: C. elegans as a model organism. Nat. Rev. Drug Discov. 5, 387-399. https://doi.org/10.1038/nrd2031 (2006).

10. Maleszka, R. The social honey bee in biomedical research: realities and expectations. Drug Discov. Today Dis. Models 12, 7-13. https://doi.org/10.1016/j.ddmod.2014.06.001 (2014).

11. Maleszka, R. Beyond Royalactin and a master inducer explanation of phenotypic plasticity in honey bees. Commun. Biol. $\mathbf{1}, 8$. https://doi.org/10.1038/s42003-017-0004-4 (2018). 
12. Fahrbach, S. E., Giray, T., Farris, S. M. \& Robinson, G. E. Expansion of the neuropil of the mushroom bodies in male honey bees is coincident with initiation of flight. Neurosci. Lett. 236, 135-138. https://doi.org/10.1016/S0304-3940(97)00772-6 (1997).

13. Fahrbach, S. E., Giray, T. \& Robinson, G. E. Volume changes in the mushroom bodies of adult honey-bee queens. Neurobiol. Learn. Mem. 63, 181-191. https://doi.org/10.1006/nlme.1995.1019 (1995).

14. Fahrbach, S. E., Moore, D., Capaldi, E. A., Farris, S. M. \& Robinson, G. E. Experience-expectant plasticity in the mushroom bodies of the honeybee. Learn. Mem. 5, 115-123 (1998).

15. Groh, C., Ahrens, D. \& Rössler, W. Environment- and age-dependent plasticity of synaptic complexes in the mushroom bodies of honeybee queens. Brain Behav. Evol. 68, 1-14, https://doi.org/10.1159/000092309 (2006).

16. Wang, Y. et al. Functional CpG methylation system in a social insect. Science N.Y. 314, 645-647. https://doi.org/10.1126/science. 1135213 (2006).

17. Miklos, G. L. G. \& Maleszka, R. Epigenomic communication systems in humans and honey bees: from molecules to behavior. Horm. Behav. 59, 399-406. https://doi.org/10.1016/j.yhbeh.2010.05.016 (2011).

18. Wedd, L. \& Maleszka, R. DNA methylation and gene regulation in honeybees: from genome-wide analyses to obligatory epialleles. Adv. Exp. Med. Biol. 945, 193-211. https://doi.org/10.1007/978-3-319-43624-1_9 (2016).

19. Allis, C. D. \& Jenuwein, T. The molecular hallmarks of epigenetic control. Nat. Rev. Genet. 17, 487-500. https://doi.org/10.1038/ nrg.2016.59 (2016).

20. Setola, V. \& Roth, B. L. Why mice are neither miniature humans nor small rats: a cautionary tale involving 5-hydroxytryptamine-6 serotonin receptor species variants. Mol. Pharmacol. 64, 1277-1278. https://doi.org/10.1124/mol.64.6.1277 (2003).

21. Maleszka, R., de Couet, H. G. \& Miklos, G. L. G. Data transferability from model organisms to human beings: insights from the functional genomics of the flightless region of Drosophila. Proc. Natl. Acad. Sci. U.S.A. 95, 3731-3736. https://doi.org/10.1073/ pnas.95.7.3731 (1998).

22. Miklos, G. L. \& Maleszka, R. Protein functions and biological contexts. Proteomics 1, 169-178. https://doi.org/10.1002/16159861(200102)1:2\%3c169::AID-PROT169\%3e3.0.CO;2-C (2001).

23. Strausfeld, N. J., Sinakevitch, I., Brown, S. M. \& Farris, S. M. Ground plan of the insect mushroom body: functional and evolutionary implications. J. Compar. Neurol. 513, 265-291. https://doi.org/10.1002/cne.21948 (2009).

24. Wojciechowski, M. et al. Insights into DNA hydroxymethylation in the honeybee from in-depth analyses of TET dioxygenase. Open Biol. https://doi.org/10.1098/rsob.140110 (2014).

25. Pastor, W. A., Aravind, L. \& Rao, A. TETonic shift: biological roles of TET proteins in DNA demethylation and transcription. Nat. Rev. Mol. Cell Biol. 14, 341-356. https://doi.org/10.1038/Nrm3589 (2013).

26. Baker, M. When antibodies mislead: the quest for validation. Nature 585, 313-314. https://doi.org/10.1038/d41586-020-02549-1 (2020).

27. Groh, C. \& Rössler, W. Caste-specific postembryonic development of primary and secondary olfactory centers in the female honeybee brain. Arthropod Struct. Dev. 37, 459-468. https://doi.org/10.1016/j.asd.2008.04.001 (2008).

28. Levine, R. B., Morton, D. B. \& Restifo, L. L. Remodeling of the insect nervous system. Curr. Opin. Neurobiol. 5, 28-35. https://doi. org/10.1016/0959-4388(95)80083-2 (1995).

29. Groh, C. \& Rössler, W. Analysis of synaptic microcircuits in the mushroom bodies of the honeybee. Insects https://doi.org/10. 3390/insects11010043 (2020).

30. Becker, N., Kucharski, R., Rössler, W. \& Maleszka, R. Age-dependent transcriptional and epigenomic responses to light exposure in the honey bee brain. FEBS Open Biol. 6, 622-639. https://doi.org/10.1002/2211-5463.12084 (2016).

31. Fahrbach, S. E. Structure of the mushroom bodies of the insect brain. Annu. Rev. Entomol. 51, 209-232. https://doi.org/10.1146/ annurev.ento.51.110104.150954 (2006).

32. Oya, S., Kohno, H., Kainoh, Y., Ono, M. \& Kubo, T. Increased complexity of mushroom body Kenyon cell subtypes in the brain is associated with behavioral evolution in hymenopteran insects. Sci. Rep. 7, 13785. https://doi.org/10.1038/s41598-017-14174-6 (2017).

33. Groh, C., Lu, Z., Meinertzhagen, I. A. \& Rössler, W. Age-related plasticity in the synaptic ultrastructure of neurons in the mushroom body calyx of the adult honeybee Apis mellifera. J. Comp. Neurol. 520, 3509-3527. https://doi.org/10.1002/cne.23102 (2012).

34. Fahrbach, S. E., Strande, J. L. \& Robinson, G. E. Neurogenesis is absent in the brains of adult honey-bees and does not explain behavioral neuroplasticity. Neurosci. Lett. 197, 145-148. https://doi.org/10.1016/0304-3940(95)11913-H (1995).

35. Muenz, T. S. et al. Neuronal plasticity in the mushroom body calyx during adult maturation in the honeybee and possible pheromonal influences. Dev. Neurobiol. 75, 1368-1384. https://doi.org/10.1002/dneu.22290 (2015).

36. Hourcade, B., Muenz, T. S., Sandoz, J. C., Rössler, W. \& Devaud, J. M. Long-term memory leads to synaptic reorganization in the mushroom bodies: a memory trace in the insect brain?. J. Neurosci. 30, 6461-6465. https://doi.org/10.1523/jneurosci.0841-10. 2010 (2010).

37. Rössler, W., Spaethe, J. \& Groh, C. Pitfalls of using confocal-microscopy based automated quantification of synaptic complexes in honeybee mushroom bodies (response to Peng and Yang 2016). Sci. Rep. 7, 9786. https://doi.org/10.1038/s41598-017-09967-8 (2017).

38. Frank, C. L. \& Tsai, L.-H. Alternative functions of core cell cycle regulators in neuronal migration, neuronal maturation, and synaptic plasticity. Neuron 62, 312-326. https://doi.org/10.1016/j.neuron.2009.03.029 (2009).

39. Werren, J. H. et al. Functional and evolutionary insights from the genomes of three parasitoid Nasonia species. Science N.Y. 327, 343-348. https://doi.org/10.1126/science.1178028327/5963/343 (2010).

40. Richards, S. et al. The genome of the model beetle and pest Tribolium castaneum. Nature 452, 949-955. https://doi.org/10.1038/ Nature06784 (2008).

41. Foret, S., Kucharski, R., Pittelkow, Y., Lockett, G. A. \& Maleszka, R. Epigenetic regulation of the honey bee transcriptome: unravelling the nature of methylated genes. BMC Genom. 10, 472. https://doi.org/10.1186/1471-2164-10-4721471-2164-10-472 (2009).

42. Foret, S. \& Maleszka, R. Function and evolution of a gene family encoding odorant binding-like proteins in a social insect, the honey bee (Apis mellifera). Genome Res. 16, 1404-1413. https://doi.org/10.1101/Gr.5075706 (2006).

43. Foret, S., Wanner, K. W. \& Maleszka, R. Chemosensory proteins in the honey bee: Insights from the annotated genome, comparative analyses and expressional profiling. Insect. Biochem. Mol. Biol. 37, 19-28. https://doi.org/10.1016/j.ibmb.2006.09.009 (2007).

44. Kucharski, R., Maleszka, J. \& Maleszka, R. A possible role of DNA methylation in functional divergence of a fast evolving duplicate gene encoding odorant binding protein 11 in the honeybee. Proc. Biol. Sci. https://doi.org/10.1098/rspb.2016.0558 (2016).

45. Hu, L. L. et al. Crystal structure of TET2-DNA complex: insight into TET-mediated 5mC oxidation. Cell 155, 1545-1555. https:// doi.org/10.1016/j.cell.2013.11.020 (2013).

\section{Acknowledgements}

We thank Robert Kucharski for his generous offer to perform analyses of the honey bee proteome and general advice on bioinformatics. Article Processing Charges were supported by the Open Access Publication Fund of the University of Würzburg. 


\section{Author contributions}

Conceptualisation and design: P.J.H., W.R., R.M.; Resources: P.J.H., W.R.; Western blotting and antibody characterisation: M.W.; Immunolabelling, microscopy and drawings: K.G., W.R.; Supervision: P.J.H., W.R.; Data evaluation: P.J.H., W.R., R.M.; Draft preparation and writing: R.M.; Writing, Review and Editing: R.M., P.J.H., W.R.; Funding acquisition: P.J.H., W.R.

\section{Funding}

Open Access funding enabled and organized by Projekt DEAL. Biotechnology and Biological Sciences Research Council, BB/L023164/1. William Harvey International Translational Research Academy, WHRI-ACADEMY. H2020 Marie Skłodowska-Curie Actions, PCOFUND-GA-2013-608765. Deutsche Forschungsgemeinschaft, INST 93/829-1. Australian Research Council, DP160103053.

\section{Competing interests}

The authors declare no competing interests.

\section{Additional information}

Supplementary Information The online version contains supplementary material available at https://doi.org/ 10.1038/s41598-021-86078-5.

Correspondence and requests for materials should be addressed to P.J.H., R.M. or W.R.

Reprints and permissions information is available at www.nature.com/reprints.

Publisher's note Springer Nature remains neutral with regard to jurisdictional claims in published maps and institutional affiliations.

(c) (1) Open Access This article is licensed under a Creative Commons Attribution 4.0 International License, which permits use, sharing, adaptation, distribution and reproduction in any medium or format, as long as you give appropriate credit to the original author(s) and the source, provide a link to the Creative Commons licence, and indicate if changes were made. The images or other third party material in this article are included in the article's Creative Commons licence, unless indicated otherwise in a credit line to the material. If material is not included in the article's Creative Commons licence and your intended use is not permitted by statutory regulation or exceeds the permitted use, you will need to obtain permission directly from the copyright holder. To view a copy of this licence, visit http://creativecommons.org/licenses/by/4.0/.

(C) The Author(s) 2021 